\title{
An Electromagnetic Sensing System Incorporating Multiple Probes and Single Antenna for Wireless Structural Health Monitoring
}

\author{
Burak Ozbey, Ayhan Altintas, \\ Hilmi Volkan Demir, and Vakur B. Erturk \\ Department of Electrical and Electronics Engineering, \\ Bilkent University, 06800, Ankara, Turkey \\ Email: ozbey@ee.bilkent.edu.tr, altintas@ee.bilkent.edu.tr
}

\author{
Ozgur Kurc \\ Department of Civil Engineering, \\ Middle East Technical University, 06800, Ankara, Turkey
}

\begin{abstract}
In this study, a wireless and passive displacement/strain sensing system is proposed for structural health monitoring (SHM). The wireless and passive interrogation of the sensing unit [a variant of a nested split-ring resonator (NSRR)] is achieved through the near-field interaction and electromagnetic coupling between the single antenna in the system and the multiple sensors called the NSRR probes. It is demonstrated that the system can acquire data from more than one NSRR probe simultaneously in a real-life scenario, where the probes are confined within concrete inside a beam, while the antenna monitors them from outside.

Index Terms-Wireless passive sensor, displacement sensor, strain sensor, nested split-ring resonator (NSRR), multi-point sensing, structural health monitoring (SHM), simply supported beam experiment.
\end{abstract}

\section{INTRODUCTION}

Structural health monitoring (SHM) is an important field which aims to prevent/detect the damage in structures by continuously or intermittently observing certain damage indicators. Due to factors such as aging, environmental effects, or sudden impacts such as an earthquake, deformation occurs in all structures, which may be threatening to human life beyond a certain point. An important damage indicator in SHM is the relative displacement and strain experienced in structural components, including reinforcing bars (rebars) embedded into concrete [1]. Majority of the commonly used strain sensing technologies in SHM (such as strain gages and fibre Bragg gratings) are wired devices, while wireless sensor networks require electrical energy for their transmitters. In order to overcome these challenges, wireless passive sensors in various geometries were proposed in literature [2]-[4]. However, the methods on which these geometries are based on have inherent problems, such as high losses for surface acoustic waves and relatively poor resolution and sensitivity for RFID. Therefore, a system is preferred between the interrogator and the sensing parts (which generally remain embedded inside the concrete) that provides a means for wireless exchange of data with a good resolution and sensitivity. In this paper, we explore the multi-point sensing capability of a wireless system based on a passive sensor geometry and a transceiver antenna. The multi-point sensing scheme offers a way to collect data from multiple sensors placed at different locations in a single frequency sweep. This capability is first verified by a controlled translation stage experiment. Then, the system is tested on a real-life simply supported beam experiment as a final stage of the operation.

\section{Sensing System Architecture}

The strain and displacement sensing structure is in nested split-ring resonator (NSRR) geometry, which incorporates a number of parallel strip pairs that are connected from one side but symmetrically separated from the other side by a gap between every pair (the structure on the bottom in Fig. 1). Each strip forms a path with the uppermost strip that is split by this gap, therefore, the whole structure can be considered as a combination of nested split-rings. The smaller size of the NSRR becomes possible via an increase in the number of metal strips, which in turn increases the overall capacitance and inductance of the structure and lowers the resonance frequency. Also, by the increased number of gaps, a high-Q resonator characteristic is achieved, providing better sensitivity and resolution. Therefore, the structure is highly convenient for sensitive strain measurements. In order to transform this structure from a strain sensor into a displacement sensor, the regular NSRR can be symmetrically split into two partitions with respect to the gap between the strips. This sensing unit, which will be referred to as the modified NSRR probe, is fastened on the structure to be monitored, e.g., a rebar, via two points at each independently moving parts. This way, the elongations or contractions on the material being monitored can directly be reflected on the probe in the form of a change of the distance between two attachment points placed at the two mechanically separated parts. The edge-to-edge distance between the two modified NSRR probe parts is denoted as $d$, as shown in Fig. 1 (top). The characteristics of the sensing system based on this modified NSRR probe geometry were previously studied and reported in [5]-[7] for different surrounding environments including free-space and various scenarios of complex media with concrete and rebars. 


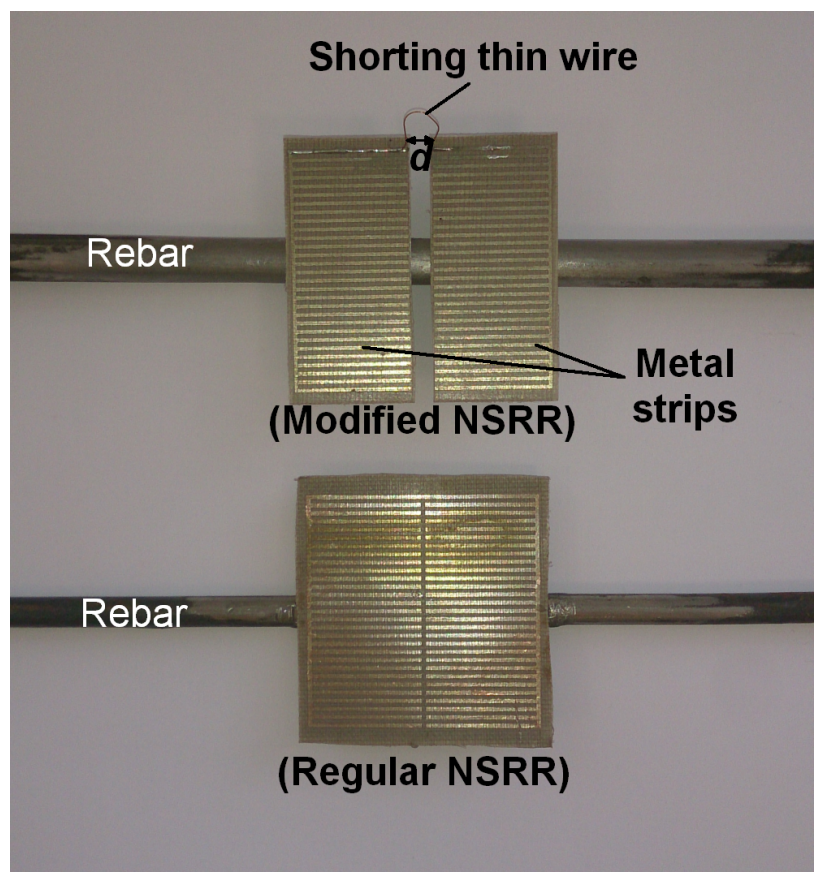

Fig. 1. Top: Modified NSRR structure for displacement/strain measurement in structural health monitoring. Bottom: Regular NSRR structure.

The underlying concept behind the sensing system is the electromagnetic coupling formed between the modified NSRR probe and the antenna. Through this electromagnetic coupling, the probe manifests itself as a local peak at its own resonance frequency on the reflection coefficient spectrum of the antenna. By monitoring this peak (frequency of the maximum point of this peak, or zero crossing of the phase, etc.), it is possible to understand the mechanical changes occurring on the modified NSRR probe, more specifically, the change of strain or displacement between the two attachment points for this particular case. In the sensing system, the antenna acts as a transceiver for the modified NSRR probe which is placed within the near-field of the antenna, transmitting the waves from a network analyzer to it and collecting the backscattered signal.

\section{Multi-Point Sensing}

It is possible to monitor more than one NSRR probe via a single antenna in the sensing system. In order to achieve it, every NSRR probe in the system should be pre-tuned to a specific resonance frequency $\left(f_{0}\right)$ and should be assigned a frequency interval $(\Delta f)$ around this resonance frequency. This frequency interval, i.e., the bandwidth, should be wide enough to capture the frequency shift experienced due to a displacement between the original attachment points. Therefore, the allocations of $f_{0}$ and $\Delta f$ should be selected carefully such that the intervals do not overlap. Then, every NSRR probe forms a communication channel with the antenna via electromagnetic coupling, from which they can be monitored in the form of a peak in the antenna reflection coefficient spectrum. By using this method, the level of displacement (or strain) induced on each of the monitored NSRR probes can be extracted in a single frequency scan of the monitoring device (e.g., a network analyzer).

In order to assign a different $f_{0}$ to every NSRR probe in the array, either the edge-to-edge initial separation between the two probe parts, $d$, or, the length of the jumper which is used to short the uppermost strip, $l$, can be varied. A change in $d$ primarily changes the capacitance of the modified NSRR, whereas a modification in $l$ primarily changes its inductance. The resonance frequency of the modified NSRR is determined via this overall capacitance and inductance, thus it strongly depends on $l$ and $d$. In Fig. 2, the result of an experiment for a two-element system $(n=2)$ is shown. Here, the initial resonance frequencies of the NSRR probes are set to 325 and $380 \mathrm{MHz}$, respectively, by choosing $l_{1}=7 \mathrm{~cm}$ and $l_{2}=3$ $\mathrm{cm}$, whereas the initial edge-to-edge distance, $d_{0}$ is 0 . The probes are excited by a microstrip single-slot antenna. Two initial local frequency peaks that correspond to each of the NSRR probes can be observed at the spectrum of the antenna reflection coefficient, as shown in Fig. 2 . In the experiment, $d_{i}$ are increased by a controlled translation stage simultaneously from 0 by $1.4 \mathrm{~mm}$, which produces a shift $(\Delta d)$ of $20 \mathrm{MHz}$ for both NSRR probes.

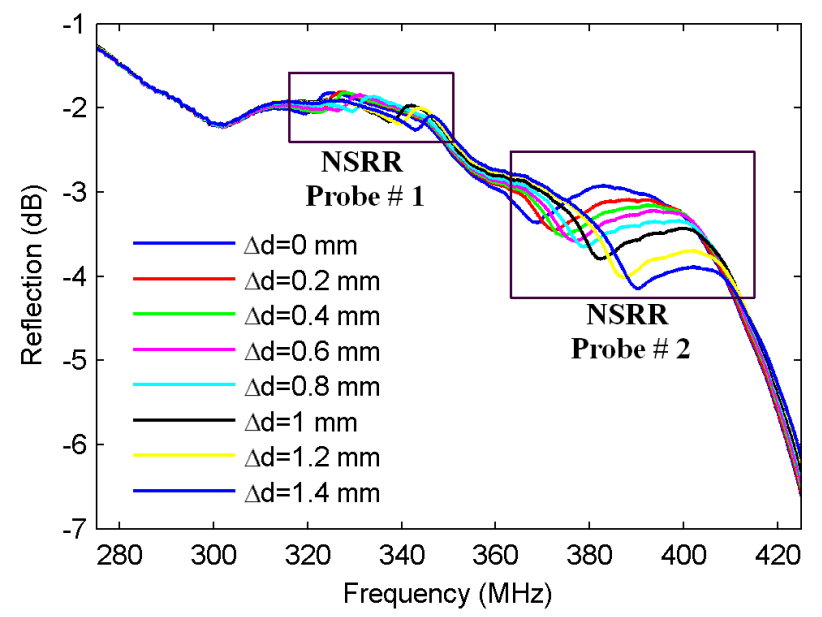

Fig. 2. The peaks at the resonance frequencies of each NSRR probe in the system $(n=2)$ when $d_{1,2}$ are increased from $d_{0}$ to $d_{0}+1.4 \mathrm{~mm}$.

\section{REAL-LIFE EXPERIMENT}

The performance of the sensing system is tested in a reallife scenario by a simply supported beam experiment, where the NSRR probes are located on the rebars inside a reinforced concrete beam [8]. Vertical force is applied from the top, which results in a tensile strain at the rebars on the bottom, which is monitored by the sensing system. The preparation of the setup is explained in Fig. 3. NSRR probes are attached on different rebars within the beam before the concrete is poured as shown in Fig. 3-a. Two of these probes have the same physical dimensions (larger geometry) and are tuned to an $f_{0}$ around $400 \mathrm{MHz}$. A protective plexiglas cover is placed before 

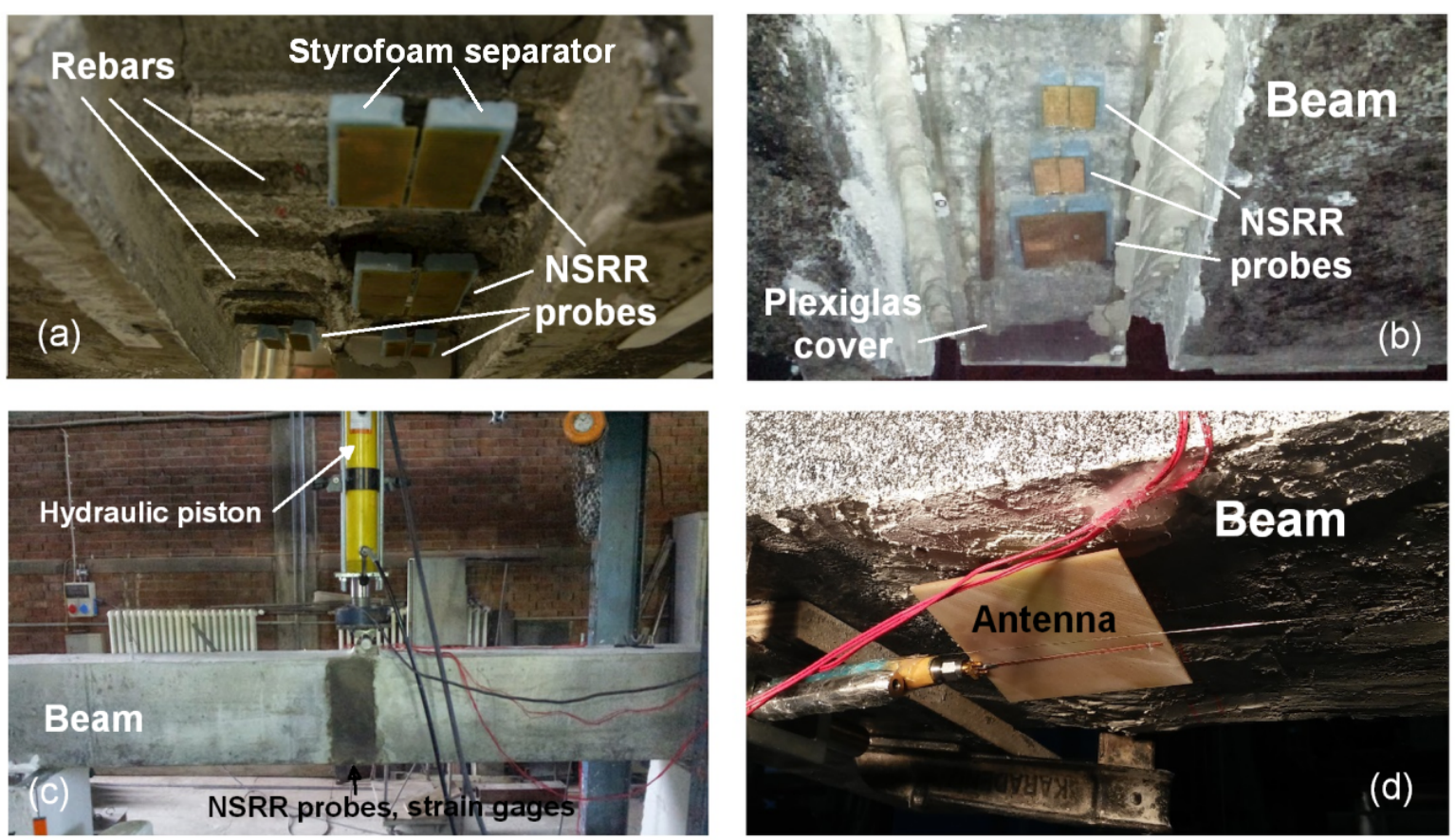

Fig. 3. Preparation of the final experiment setup: a) The arrangement of the NSRR sensors, b) Placement of the protective plexiglas cover, c) The final simply supported beam experiment setup, d) Antenna monitoring the sensors from outside the bottom of the beam.

the probes as shown in Fig. 3-b in order to ensure that there is no contact between the probes and the concrete. Finally, a thin plaster layer is placed on the plexiglas cover, closing the beam up. The final form of the setup is shown in Fig. 3-c. The strain/displacement induced on the NSRR probes can be monitored via an antenna placed outside the beam (see Fig. 3d). During the experiment, both the strain gages connected to a data acquisition system, and the NSRR probes record data at the same time instants. These data will be presented and discussed in detail during the presentation.

\section{CONCLUSION}

In this study, multi-point sensing capability via a wireless and passive displacement/strain sensing system is proposed. The system incorporates sensing units in a modified NSRR geometry and a transceiver antenna. The electromagnetic coupling between the antenna and the probes separately enables the monitoring of each NSRR probe in the system without getting affected by each other. The multi-point sensing technique is verified by a proof-of-concept translation stage experiment for three NSRR probes. Finally, the system is tested in a reallife simply supported beam experiment, whose results will be discussed during the presentation.

\section{REFERENCES}

[1] A. Mita and S. Takhira, "A smart sensor using mechanical memory for structural health monitoring of a damage-controlled building," Smart Mater. Struct., vol. 12, no. 2, 2003.
[2] D. Thomson, D. Card, and G. Bridges, "RF Cavity Passive Wireless Sensors With Time-Domain Gating-Based Interrogation for SHM of Civil Structures," IEEE Sensors Journal, vol. 9, no. 11, pp. 1430-1438, Nov. 2009

[3] S.-D. Jang and J. Kim, "Passive wireless structural health monitoring sensor made with a flexible planar dipole antenna," Smart Mater. Struct., vol. 21, no. 2, p. 027001, 2012.

[4] C. Paggi, C. Occhiuzzi, and G. Marrocco, "Sub-Millimeter displacement sensing by passive UHF RFID antennas," IEEE Trans. Microw. Theory Tech., vol. 62, no. 2, pp. 905-912, Feb. 2014.

[5] B. Ozbey, E. Unal, H. Ertugrul, O. Kurc, C. M. Puttlitz, V. B. Erturk, A. Altintas, and H. V. Demir, "Wireless displacement sensing enabled by metamaterial probes for remote structural health monitoring," Sensors, vol. 14, no. 1, pp. 1691-1704, Jan. 2014.

[6] B. Ozbey, H. V. Demir, O. Kurc, V. B. Erturk, and A. Altintas, "Wireless measurement of elastic and plastic deformation by a metamaterial-based sensor," Sensors, vol. 14, no. 10, pp. 19609-19621, Oct. 2014.

[7] B. Ozbey, H. V. Demir, O. Kurc, V. B. Ertürk, and A. Altintas, "Wireless sensing in complex electromagnetic media: Construction materials and structural monitoring," IEEE Sensors J., vol. 15, no. 10, pp. 5545-5554, 2015.

[8] B. Ozbey, V. B. Erturk, H. V. Demir, A. Altintas, and O. Kurc, "A Wireless Passive Sensing System for Displacement/Strain Measurement in Reinforced Concrete Members," Sensors, vol. 16, no. 4, p. 496, Apr. 2016. 